\title{
SCIENTIFIC REPORTS

\section{OPEN Concurrent administration of IFNo14 and cART in TKO-BLT mice enhances suppression of HIV-1 viremia but does not eliminate the latent reservoir}

\author{
Kathrin Sutter $\mathbb{D}^{1,4}$, Kerry J. Lavender $\mathbb{D}^{2,3,4}$, Ronald J. Messer ${ }^{2}$, Marek Widera ${ }^{1}$, \\ Katie Williams ${ }^{2}$, Brent Race ${ }^{2}$, Kim J. Hasenkrug ${ }^{2 *}$ \& Ulf Dittmer ${ }^{1 *}$
}

Combination antiretroviral therapy (CART) prevents HIV-1 replication but does not eliminate the latent reservoir and cure the infection. Type I interferons (IFN) mediate antiviral effects through different mechanisms than CART. We previously showed that IFN 14 is the most potent IFN $\alpha$ subtype against HIV-1 and that it can significantly reduce the HIV-1 proviral reservoir. This study sought to determine whether combining CART with IFNo14 therapy would produce greater reductions in HIV-1 viral and proviral loads than ART alone. Immunodeficient Rag2 $^{-I-} \gamma_{c}{ }^{-1-} \mathrm{CD} 47^{-1-} \mathrm{C} 57 \mathrm{BL} / 6$ mice were humanized by the BLT method, infected with HIV-1 $1_{\mathrm{JR}-\mathrm{CSF}}$ and the in vivo efficacy of $\mathrm{CART}$ was compared with combined CART/IFN 14 therapy. Infection was allowed to establish for 6 weeks prior to 4 weeks of treatment with oral cART either with or without IFNo14. Plasma viral RNA and splenic $\mathrm{CD4}^{+} \mathrm{T}$ cell viral DNA levels were measured immediately after treatment and after 2 weeks of therapy interruption. Augmentation of CART with IFN $\alpha 14$ resulted in significantly enhanced suppression of HIV-1 plasma viremia. However, no significant reduction in total viral DNA was detectable. Furthermore, virus rebounded after treatment interruption to similar levels in both groups. Thus, augmentation of CART with IFNQ14 resulted in a more pronounced reduction of HIV viremia levels over CART alone, but the effect was not potent enough to be detected at the viral DNA level or to prevent virus rebound following therapy interruption in immune system-humanized mice.

The major obstacle in developing a sterilizing or functional cure for HIV-1 infection is the presence of a reservoir of latently infected cells. Although combination antiretroviral therapy (cART) can efficiently interfere with active virus replication and suppress viremia to very low or undetectable levels, it cannot purge the reservoir of latently infected cells. Thus, cART interruption ultimately leads to reactivation of the reservoir and viremia. Many cure strategies aim to harness aspects of the host immune response to clear latently infected cells or to induce immunological control upon treatment interruption. The testing and development of such strategies are facilitated by the use of small animal models, such as the triple knockout bone marrow-liver-thymus (TKO-BLT)-humanized mouse model ${ }^{1,2}$. TKO-BLT mice become reconstituted with high levels of multi-lineage human hematopoietic cells, are susceptible to HIV-1 infection, and develop hallmarks of human HIV-1 infection such as hyper-immune activation and $\mathrm{CD} 4^{+} \mathrm{T}$ cell depletion. They are also resistant to graft versus host disease so they can be studied relatively long-term in the absence of an underlying immunopathological condition ${ }^{1,2}$. Like other BLT models, TKO-BLT mice can be infected with HIV-1 and treated with ART to establish latent HIV-1 infection, which rapidly recrudesces after treatment interruption ${ }^{3,4}$. Importantly for cure studies that rely on endogenous immunity, BLT mice also have functional immune responses including HIV-1-specific responses ${ }^{1,5,6}$.

${ }^{1}$ Institute for Virology, University Hospital Essen, University of Duisburg-Essen, 45122, Essen, Germany. ${ }^{2}$ Laboratory of Persistent Viral Diseases, Rocky Mountain Laboratories, NIAID, NIH, Hamilton, MT, USA. ${ }^{3}$ Present address: Department of Biochemistry, Microbiology \& Immunology, College of Medicine, University of Saskatchewan, Saskatoon, SK, Canada. ${ }^{4}$ These authors contributed equally: Kathrin Sutter and Kerry J. Lavender. *email: khasenkrug@nih.gov; ulf.dittmer@uni-due.de 
We previously showed that a specific interferon alpha (IFN $\alpha$ ) subtype, IFN $\alpha 14$, mediated superior suppression of HIV-1 infection compared to other subtypes in TKO-BLT mice ${ }^{7}$. Interestingly, both intrinsic and innate immunity were associated with the anti-HIV-1 activity mediated by IFN $\alpha 14$. For example, increased levels of signature APOBEC3G mutations were found in proviral DNA, potentially reducing the replicative fitness of the reservoir. Furthermore, IFN $\alpha 14$ specifically activated NK cells, which may have eliminated virally infected cells. It was therefore an attractive strategy to administer IFN $\alpha 14$ in conjunction with cART to harness their combined and divergent mechanisms of action in order to potentially produce reductions in the size and/or fitness of the latent reservoir. The use of IFN $\alpha$ in the treatment of HIV-1 or as a component of a cure strategy is controversial, particularly due to recent studies blocking the IFN $\alpha / \beta$ receptor (IFNAR) in HIV-1 infected humanized mice. One study using IFNAR blockade demonstrated that, despite having antiviral effects, IFNAR signaling may drive $\mathrm{CD} 4^{+} \mathrm{T}$ cell depletion and dysfunction of $\mathrm{CD} 4^{+}$and $\mathrm{CD} 8^{+} \mathrm{T}$ cells during chronic infection ${ }^{8}$. Additionally, Zhen and colleagues reported that ART combined with IFNAR blockade in HIV-1 infected BLT mice decreased plasma RNA levels as well as numbers of latently infected cells ${ }^{9}$. In contrast to these studies that block all type I IFN-mediated effects, we and others have reported that specific IFN $\alpha$ subtypes can mediate beneficial effects in HIV-1 infected humanized mice ${ }^{7,10}$. This discrepancy in methodology is worth consideration as seminal work in the chronic LCMV mouse model has shown that despite signaling through the same receptor, only IFN $\beta$ and not IFN $\alpha$ impaired antiviral immunity and supported persistent infection ${ }^{11}$. Thus, subtype specific IFN $\alpha$ treatment of HIV-1 infection remains a viable therapeutic option and is worth pursuing, as evidenced by continued HIV-1 clinical trials aiming to harness the potent effects of IFN $\alpha$ on the HIV-1 reservoir. Such studies in HIV-1 infected subjects are currently underway using the IFN 22 subtype (NCT02227277). Unfortunately, the use of the IFN $\alpha 2$ subtype is based on its current approval status for clinical use against hepatitis viruses, but it has mostly been shown to have low antiviral activity against HIV-1 in vitro ${ }^{12}$ and in vivo ${ }^{7,13-17}$. In HIV-1 patients co-infected with HCV, short-term treatment with cART and IFN $\alpha 2$ reduced HIV-1 expression and decreased CD4+ T cell activation ${ }^{18}$. In all the studies published so far, the most potent human IFN $\alpha$ subtype against HIV-1 has been IFN $\alpha 14^{7,10,12}$. We therefore used the TKO-BLT human immune system mouse model to assess whether the addition of IFN $\alpha 14$ therapy to cART during suppression of viral replication would produce a smaller and/or less replication competent latent HIV reservoir.

\section{Materials and Methods}

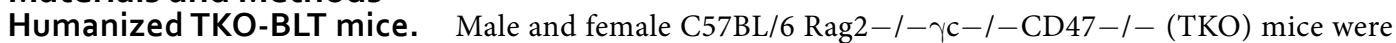
humanized using the bone marrow, liver, thymus (BLT) method as previously described [2]. Animals were housed under specific pathogen-free conditions. All animal studies were performed under an AAALAC-accredited Rocky Mountain Laboratories, National Institute of Allergy and Infectious Diseases, National Institutes of Health (USA) Institutional Animal Care and Use Committee-approved animal study protocol in accordance with the regulations and guidelines of the Animal Care and Use Committee of the Rocky Mountain Laboratories, NIAID, NIH. The study included provisions to ensure proper anesthesia during procedures, pain relief medication during recovery and daily health monitoring. The use of donated anonymous fetal tissue biospecimens for the research described in this paper was approved by the National Institutes of Health (NIH) Office of Human Subjects Research Protection (OHSRP Project ID\# P194542; Ref. 526739). Donor tissues for humanization were obtained with informed consent following all guidelines and regulations of NIH and the Office of Human Subjects Research Protection. All research involving human fetal tissue was completed before June 5, 2019.

HIV-1 challenge, CART and CART plus IFN $\alpha 14$ treatment. R5-tropic HIV-1 $1_{\text {JR-CSF }}$ stocks were prepared and inoculated intraperitoneally as previously described ${ }^{1}$. TZM-bl (JC53-bl) reporter cells (NIH AIDS Research and Reference Reagent Program from Drs John Kappes and Xiaoyun Wu and Tranzyme, Inc.) were used to determine stock concentrations. Infected mice were assigned to groups with similar mean p24 antigenemia at 6 weeks post infection (wpi). Starting at 6 weeks post infection, mice were free-fed with cART-supplemented tenofovir (TDF; Gilead Sciences, Foster City, CA, USA), emtricitabin (FTC; Gilead Sciences) and raltegravir (RAL; Merck \& Co., Kenilworth, NJ, USA) mouse chow as previously described ${ }^{4}$. One group of mice additionally received intraperitoneal injections of $1.5 \times 10^{5} \mathrm{U} /$ mouse IFN $\alpha 14$ after 6 weeks of infection and subsequently at $24 \mathrm{~h}$ intervals for 4 weeks.

Quantification of HIV-1 RNA and DNA. Plasma HIV RNA was isolated with the QIAamp Viral RNA Kit (Qiagen, Hilden, Germany) and quantified using the Abbott RealTime HIV-1 m2000 test system as described by the manufacturers. By using an input volume of $100 \mu \mathrm{l}$ plasma the detection limit was $300 \mathrm{copies} / \mathrm{ml}$. Total HIV-1 DNA was quantified performing a pre-PCR followed by a probe-based real-time PCR approach as described previously ${ }^{19,20}$. Briefly, genomic DNA was isolated from splenic CD4 ${ }^{+}$cells using QIAamp DNA Mini Kit (Qiagen). CD3 and HIV-1 DNA sequences were pre-amplified by carrying out 12 cycling steps in a TProfessional TRIO Thermocycler (Biometra, Goettingen, Germany). The pre-PCR amplicons were diluted and subjected to quantitative real-time PCR analysis using the Rotor-Gene Probe PCR Kit (Qiagen) performed in a Rotor-Gene Q instrument (Qiagen). TaqMan probes for CD3 and HIV-1-DNA quantification were dually labelled with YAK-BHQ-1 and 6-FAM-BHQ-1, respectively. Standard curves were generated with plasmid DNA templates harboring the corresponding amplicon regions or genomic DNA of HIV-1 LTR-harbouring cells for the quantification of integrated provirus $^{20}$. Determinations of HIV infection levels in plasma for group assignments were done by p24 ELISA (Advanced Bioscience Laboratories, Rockville MD).

Recombinant IFNo14. Production of human IFN $\alpha 14$ protein was previously described ${ }^{7}$ and endotoxin levels were less than 0.0025 endotoxin units (EU)/ml (ToxinSensor; Genscript, Piscataway, NJ). The biological activity of the recombinantly produced IFN $\alpha 14$ was measured with an IFN-stimulated response element (ISRE) based 
A
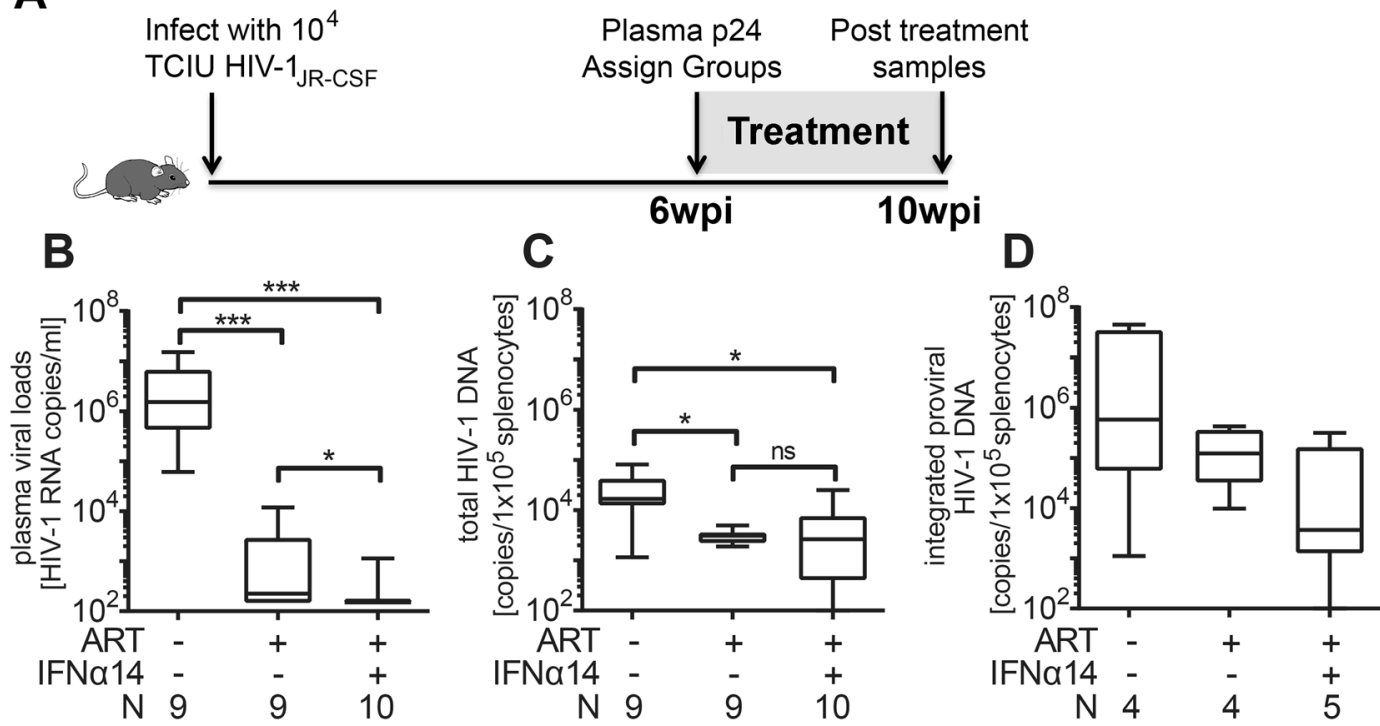

Figure 1. Addition of IFN $\alpha 14$ to cART therapy for 4 weeks significantly reduces plasma viral RNA but does not decrease cellular viral DNA levels. (A) Scheme of the experimental timeline. Mice were inoculated i.p. with $1 \times 10^{4} \mathrm{TCIUs}$ of HIV- $1_{\mathrm{JR}-\mathrm{CSF}}$ and infection was allowed to progress for 6 weeks. At 6 wpi p24-CA levels were determined and mice were assigned to groups with comparable HIV-1 antigen loads. Mice were either freefed with cART chow $(n=18)$, given cART chow plus daily i.p. injections of $1.5 \times 10^{5}$ units of IFN $\alpha 14(n=20)$ or left untreated $(\mathrm{n}=18)$ for 4 weeks (10wpi). (B) Levels of HIV-1 viral RNA in plasma were measured directly after analytical treatment interruption (10wpi). Student's t test; $*<0.05$, ns $=$ not significant. (C) Levels of total HIV-1 viral DNA and (D) levels of integrated proviral HIV-1 DNA from CD4 ${ }^{+}$enriched splenocytes were determined (10wpi). One-way ANOVA with Tukey's post-test (untreated n=9, ART n =9, ART + IFN $\alpha 14$ $\mathrm{n}=10$ ). Box and whisker plots depict means with standard deviations and ranges. $\mathrm{N}=$ numbers of mice investigated.

reporter assay and normalized to commercially available IFNo14 (PBL Assay Science, Piscataway Township, NJ, USA), as described elsewhere ${ }^{7}$.

\section{Results and Discussion}

Infection of humanized TKO-BLT mice ${ }^{1,2}$ was done by intraperitoneal inoculation of $10^{4}$ tissue culture infectious

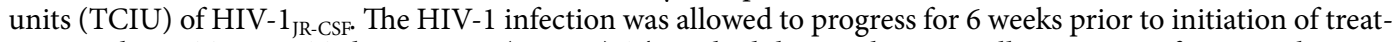
ment with cART or cART plus IFN $\alpha 14$ (Fig. 1A). This schedule was chosen to allow HIV-1 infection to become well established and to represent a reasonable time frame relative to human patients seeking clinical treatment for symptomatic HIV-1 infections. Since the dosage of IFN $\alpha$ that can be used clinically is limited by undesirable side effects $^{21}$, we used the mouse equivalent ${ }^{22}$ units of IFN $\alpha 14$ used to treat melanoma patients with IFN $\alpha 2^{23}$, which was previously shown to suppress HIV-1 replication and reduce proviral loads in TKO-BLT mice 7 .

At 6 weeks post infection all mice were tested for plasma p24 levels and placed in three groups with closely equivalent mean HIV-1 p24 antigen levels. Virus suppression was initiated in two of the groups using free feeding of drugs incorporated into mouse chow. One of the cART groups was also treated by daily injections of IFN 14 for 4 weeks. One day after the cessation of the 4 week treatment period, all mice were analyzed for plasma viral RNA (Fig. 1B). The experiment was repeated in two independent cohorts of mice that gave very similar results so the data were combined for analysis. Results showed that the addition of IFN $\alpha 14$ therapy to cART resulted in a statistically significant 8-fold reduction in plasma virus RNA levels compared to cART alone (Fig. 1B).

In HIV-1 infected humans, cART alone cannot purge the latent DNA reservoir but has been reported to reduce cellular HIV-1 DNA levels over the first year of therapy ${ }^{24}$. To analyze DNA levels in the mice following cART, a subset of mice from each group was euthanized to harvest spleens for isolation of CD4 ${ }^{+} \mathrm{T}$ cell DNA. Both of the treatment arms produced a statistically significant reduction in the mean HIV-1 DNA levels compared to the untreated control group, but the addition of IFN $\alpha 14$ therapy to cART did not result in any further reduction in total splenic viral DNA (Fig. 1C). The viral reservoir size is better described by measuring integrated HIV provirus levels than total HIV DNA. Unfortunately, we could perform such assays for only $4-5$ mice per group due to sample size limitations. ART treatment alone (median: $1.2 \times 10^{5}$ ) did not significantly change copy numbers of integrated HIV DNA compared to HIV-infected, untreated controls $\left(5.8 \times 10^{5}\right)$ (Fig. 1D). In contrast, the combination therapy of ART and IFN $\alpha 14$ reduced proviral DNA levels by more than two logs $\left(3.6 \times 10^{3}\right)$. However, due to the small sample size this difference was not statistically significant. The data nevertheless suggested that the combination therapy might affect the viral reservoir size of HIV.

Because many proviruses may be defective, the HIV-1 DNA content does not reflect the number of transcriptionally active and/or virus-producing cells. Additionally, we and others ${ }^{7,12}$ have previously shown that IFN $\alpha 14$ induces increased levels of APOBEC3G signature mutations in HIV-1 that could affect viral fitness without 
A
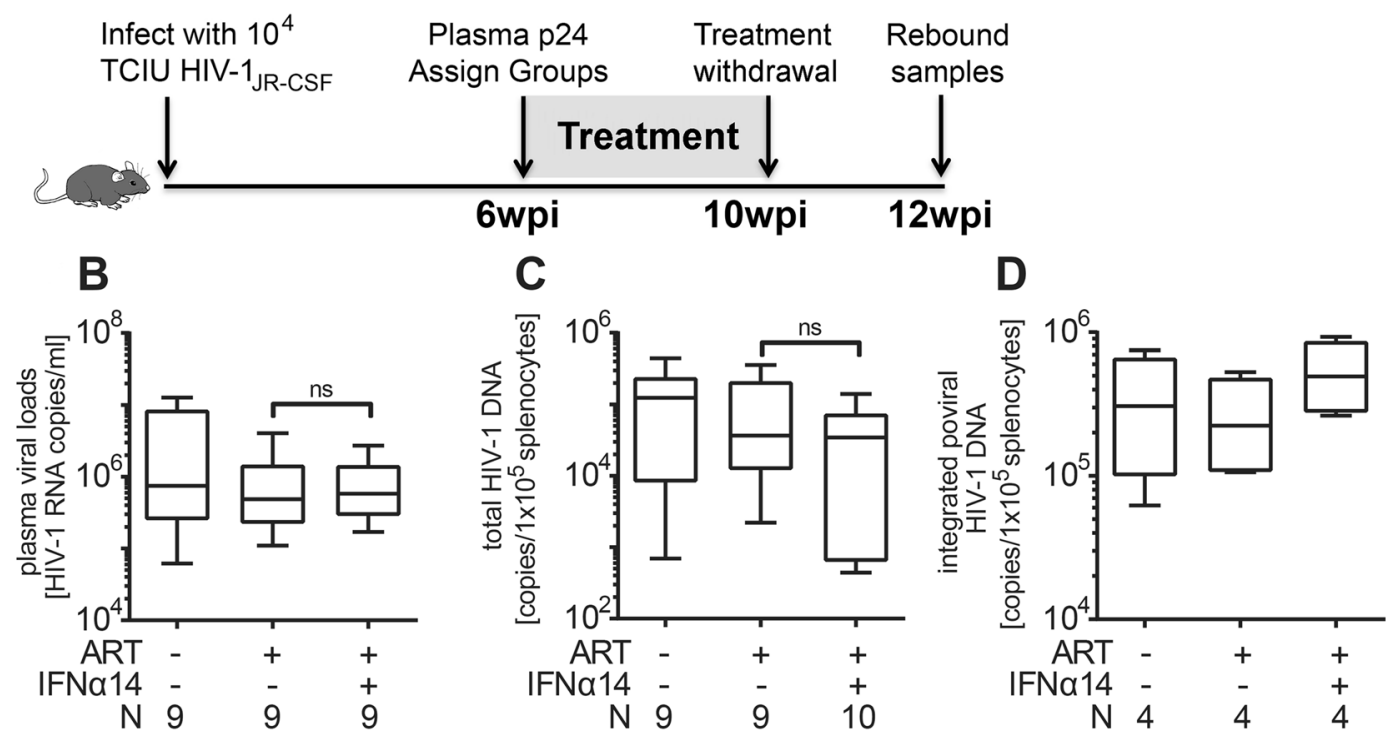

Figure 2. HIV-1 viremia and viral recrudesce in cART or cART plus IFN $\alpha 14$ treated mice after 2 weeks of treatment withdrawal. (A) Schematic drawing of the experimental timeline. Mice were inoculated i.p with $1 \times 10^{4}$ TCIUs of HIV-1 $1_{\text {IR-CSF }}$ and infection was allowed to progress for 6 weeks. At 6 wpi mice were either freefed with cART chow, given cART chow plus daily i.p. injections of IFN 14 or left untreated for 4 weeks. At 10wpi all treatment was discontinued (analytical treatment interruption) for additional 2 weeks prior to sample collection at 12wpi. (B) Levels of HIV-1 viral RNA in plasma (untreated $n=9$, ART $n=9$, ART + IFN $\alpha 14$ $\mathrm{n}=9$ ) and (C) levels of total HIV-1 viral DNA and (D) levels of integrated proviral HIV-1 DNA in CD4 ${ }^{+}$ enriched splenocytes (untreated $n=9, A R T n=9, A R T+I F N \alpha 14 n=10$ ) were analysed. Box and whisker plots depict means with standard deviations and ranges. One-way ANOVA with Tukey's post-test; ns = not significant. $\mathrm{N}=$ numbers of mice investigated.

altering total DNA levels. Thus, we used analytical cART interruption to evaluate virus rebound, looking for potential IFN $\alpha 14$-associated reductions or delays in viral recrudescence. cART was discontinued for 2 weeks after treatment (Fig. 2A) and the animals were analyzed for viral RNA levels (Fig. 2B). No significant differences between the cART-only and the cART plus IFN $\alpha 14$ groups were observed. At 2 weeks post-cART interruption, the HIV-1 viral RNA levels (Fig. 2B) as well as total DNA (Fig. 2C) and integrated proviral DNA levels (Fig. 2D) were almost as high as in the untreated controls and not significantly different from each other. Thus, addition of IFN $\alpha 14$ therapy to cART during established HIV-1 infection provided no observable long-lasting benefit in preventing virus rebound following therapy interruption.

The current results reveal that cART plus IFN $\alpha 14$ therapy may be more efficacious than cART alone in reducing HIV-1 plasma viral loads (Fig. 1B). Thus, it could be used for patients who are refractory to full virus suppression from cART alone, patients who develop resistance mutations affecting multiple ART drug classes, or during periods of cART interruption. However, the concurrent administration of IFN $\alpha 14$ and cART during the induction of HIV-1 latency in humanized TKO-BLT mice at the doses and treatment duration tested, had no effect on the replication competent HIV-1 reservoir as evidenced by no reduction in the amount of cellular viral DNA, and no reduction or delay in viral recrudescence upon treatment interruption. It is quite possible that cART suppression of virus replication might have limited the antiviral effect of the IFN $\alpha 14$ in vivo if active viral replication is required for IFN $\alpha 14$ to mediate its effect on the DNA reservoir. This could occur, for example, if IFN $\alpha 14$-activated NK cells could not recognize and kill latently infected cells. Since these experiments were performed in humanized mice rather than humans, there may have been anomalous interactions between IFN $\alpha 14$ and mouse cells resulting in a failure of the combination therapy in effecting the viral reservoir. For example, IFN $\alpha 14$ has been shown to activate NK cells in HIV infections, but the levels of NK cells in TKO-BLT-humanized mice are not as high as they are in humans and may not be able to exert a sufficient antiviral effect ${ }^{7}$. It is also possible that testing of additional mice might produce statistically significant effects especially with longer treatment schedules or in combination with latency reversal drugs. Although these negative results are not encouraging for HIV cure, IFN $\alpha$ subtypes remain interesting drug candidates for HIV-1 therapy because of their multifunctional antiviral properties, including the possible re-activation of latent HIV, the induction of HIV restriction factors, protection of uninfected host cells against HIV infection (antiviral state), and immune-stimulatory activities.

Received: 26 June 2019; Accepted: 15 November 2019;

Published online: 02 December 2019 


\section{References}

1. Lavender, K. J. et al. BLT-humanized C57BL/6 Rag2-/- gammac-/- CD47-/- mice are resistant to GVHD and develop B- and T-cell immunity to HIV infection. Blood 122, 4013-4020, https://doi.org/10.1182/blood-2013-06-506949 (2013).

2. Lavender, K. J., Messer, R. J., Race, B. \& Hasenkrug, K. J. Production of bone marrow, liver, thymus (BLT) humanized mice on the C57BL/6 Rag2(-/-)gammac(-/-)CD47(-/-) background. Journal of immunological methods 407, 127-134, https://doi. org/10.1016/j.jim.2014.04.008 (2014).

3. Denton, P. W. et al. Generation of HIV Latency in Humanized BLT Mice. Journal of virology 86, 630-634, https://doi.org/10.1128/ Jvi.06120-11 (2012).

4. Halper-Stromberg, A. et al. Broadly neutralizing antibodies and viral inducers decrease rebound from HIV-1 latent reservoirs in humanized mice. Cell 158, 989-999, https://doi.org/10.1016/j.cell.2014.07.043 (2014).

5. Dudek, T. E. et al. Rapid evolution of HIV-1 to functional CD8(+) T cell responses in humanized BLT mice. Sci Transl Med 4, 143ra198, https://doi.org/10.1126/scitranslmed.3003984 (2012).

6. Brainard, D. M. et al. Induction of robust cellular and humoral virus-specific adaptive immune responses in human immunodeficiency virus-infected humanized BLT mice. Journal of virology 83, 7305-7321 (2009).

7. Lavender, K. J. et al. Interferon Alpha Subtype-Specific Suppression of HIV-1 Infection In Vivo. Journal of virology 90, 6001-6013, https://doi.org/10.1128/JVI.00451-16 (2016).

8. Cheng, L. et al. Blocking type I interferon signaling enhances T cell recovery and reduces HIV-1 reservoirs. The Journal of clinical investigation 127, 269-279, https://doi.org/10.1172/JCI90745 (2017).

9. Zhen, A. et al. Targeting type I interferon-mediated activation restores immune function in chronic HIV infection. The Journal of clinical investigation 127, 260-268, https://doi.org/10.1172/JCI89488 (2017).

10. Abraham, S. et al. Gene therapy with plasmids encoding IFN-beta or IFN-alpha14 confers long-term resistance to HIV-1 in humanized mice. Oncotarget, https://doi.org/10.18632/oncotarget.12512 (2016).

11. Ng, C. T. et al. Blockade of interferon Beta, but not interferon alpha, signaling controls persistent viral infection. Cell host \& microbe 17, 653-661, https://doi.org/10.1016/j.chom.2015.04.005 (2015).

12. Harper, M. S. et al. Interferon-alpha Subtypes in an Ex Vivo Model of Acute HIV-1 Infection: Expression, Potency and Effector Mechanisms. PLoS pathogens 11, e1005254, https://doi.org/10.1371/journal.ppat.1005254 (2015).

13. Asmuth, D. M. et al. Safety, tolerability, and mechanisms of antiretroviral activity of pegylated interferon Alfa-2a in HIV-1monoinfected participants: a phase II clinical trial. The Journal of infectious diseases 201, 1686-1696, https://doi.org/10.1086/652420 (2010).

14. Hubbard, J. J. et al. Host gene expression changes correlating with anti-HIV-1 effects in human subjects after treatment with peginterferon Alfa-2a. The Journal of infectious diseases 205, 1443-1447, https://doi.org/10.1093/infdis/jis211 (2012).

15. Katsounas, A. et al. Differential Specificity of Interferon-alpha Inducible Gene Expression in Association with Human Immunodeficiency Virus and Hepatitis C Virus Levels and Declines in vivo. J AIDS Clin Res 6, https://doi.org/10.4172/21556113.1000410 (2015).

16. Azzoni, L. et al. Pegylated Interferon alfa-2a monotherapy results in suppression of HIV type 1 replication and decreased cellassociated HIV DNA integration. The Journal of infectious diseases 207, 213-222, https://doi.org/10.1093/infdis/jis663 (2013).

17. Abdel-Mohsen, M. et al. Effects of alpha interferon treatment on intrinsic anti-HIV-1 immunity in vivo. Journal of virology 88, 763-767, https://doi.org/10.1128/JVI.02687-13 (2014).

18. Moron-Lopez, S. et al. Short-term treatment with IFNalpha diminishes expression of HIV-1 and reduces CD4+ T-cell activation in HIV/HCV-coinfected patients on antiretroviral therapy. The Journal of infectious diseases, https://doi.org/10.1093/infdis/jiv521 (2015).

19. Vandergeeten, C. et al. Cross-clade ultrasensitive PCR-based assays to measure HIV persistence in large-cohort studies. J Virol 88, 12385-12396, https://doi.org/10.1128/JVI.00609-14 (2014).

20. Widera, M. et al. HIV-1 persistent viremia is frequently followed by episodes of low-level viremia. Med Microbiol Immunol 206, 203-215, https://doi.org/10.1007/s00430-017-0494-1 (2017).

21. Pestka, S. The interferons: 50 years after their discovery, there is much more to learn. The Journal of biological chemistry 282, 20047-20051, https://doi.org/10.1074/jbc.R700004200 (2007).

22. Reagan-Shaw, S., Nihal, M. \& Ahmad, N. Dose translation from animal to human studies revisited. FASEB journal: official publication of the Federation of American Societies for Experimental Biology 22,659-661, https://doi.org/10.1096/fj.07-9574LSF (2008).

23. Daud, A. et al. Management of pegylated interferon alpha toxicity in adjuvant therapy of melanoma. Expert opinion on biological therapy 12, 1087-1099, https://doi.org/10.1517/14712598.2012.694421 (2012).

24. Deeks, S. G., Lewin, S. R. \& Havlir, D. V. The end of AIDS: HIV infection as a chronic disease. Lancet 382, 1525-1533, https://doi. org/10.1016/S0140-6736(13)61809-7 (2013).

\section{Acknowledgements}

We thank Barbara Bleekmann for excellent technical assistance. This research was supported in part by the Intramural Research Program of the National Institute of Allergy and Infectious Diseases, National Institutes of Health, USA. KS and UD were supported by a grant from the German Research Association (DFG DI 714/18-1/2 and GI 974/1-1/2).

\section{Author contributions}

K.S. and K.J.L. designed experiments, performed experiments, analyzed and interpreted data and wrote the paper; R.J.M., M.W., K.W. and B.R. performed experiments, K.J.H. and U.D. conceived experiments, supervised personnel, analyzed and interpreted data and wrote the paper.

\section{Competing interests}

The authors declare no competing interests.

\section{Additional information}

Correspondence and requests for materials should be addressed to K.J.H. or U.D.

Reprints and permissions information is available at www.nature.com/reprints.

Publisher's note Springer Nature remains neutral with regard to jurisdictional claims in published maps and institutional affiliations. 
(c) (i) Open Access This article is licensed under a Creative Commons Attribution 4.0 International License, which permits use, sharing, adaptation, distribution and reproduction in any medium or format, as long as you give appropriate credit to the original author(s) and the source, provide a link to the Creative Commons license, and indicate if changes were made. The images or other third party material in this article are included in the article's Creative Commons license, unless indicated otherwise in a credit line to the material. If material is not included in the article's Creative Commons license and your intended use is not permitted by statutory regulation or exceeds the permitted use, you will need to obtain permission directly from the copyright holder. To view a copy of this license, visit http://creativecommons.org/licenses/by/4.0/.

(C) The Author(s) 2019 